\title{
Abordagem Exploratória do Emprego das Técnicas de Análise de Regressão e Análise Conjunta em Estudos do Comportamento do Consumidor
}

\author{
Exploratory Approach to the use of Techniques of Regression Analysis \\ and Pooled Analysis in Studies of Consumer Behavior
}

\author{
Estudio Exploratorio sobre la Utilización de Técnicas de Análisis de Regresión \\ $y$ de Análisis Conjunto en los Estudios del Comportamiento del Consumidor
}

\author{
Maria Aparecida Gouvêa ${ }^{1}$ \\ Leandro Campi Prearo ${ }^{2}$ \\ Maria do Carmo Romeiro ${ }^{3}$
}

Recebido em 04 de fevereiro de 2010 / Aprovado em 19 de agosto de 2010

Editor Responsável: João Maurício Gama Boaventura, Dr.

Processo de Avaliação: Double Blind Review

\section{RESUMO}

Este artigo faz parte de um amplo estudo de avaliação da adequação no uso de técnicas estatísticas multivariadas em teses e dissertações de algumas instituiçôes de ensino superior na área de marketing na temática do comportamento do consumidor entre 1997 e 2006 . Neste artigo são focalizadas as técnicas de análise de regressão e análise conjunta, duas técnicas com grande potencial de uso em estudos de marketing. Foi objetivo desse trabalho a análise da adequação do emprego destas técnicas às necessidades dos problemas de pesquisa apresentados nas teses e dissertaçóes e a aferição do nível de acerto no atendimento de suas premissas. De forma geral, os resultados sugerem a necessidade de um aumento do comprometimento dos pesquisadores na verificação de todos os preceitos teóricos de aplicação destas técnicas classificadas na categoria de investigação de dependência entre variáveis.

Palavras-chave: Métodos quantitativos. Análise de regressão múltipla. Análise conjunta. Comportamento do consumidor.

1. Doutora e Professora da Universidade de São Paulo, Faculdade de Economia, Administração e Contabilidade - FEA/USP [magouvea@usp.br]

Endereço da autora: Av. Prof. Luciano Gualberto, 908, São Paulo - SP Cep. 05508-900 - Brasil

2. Doutorando em Administração na Universidade de São Paulo, Faculdade de Economia, Administração e Contabilidade. Professor da Universidade Municipal de São Caetano do Sul - USCS [leandro.prearo@uscs.edu.br] Endereço do autor: Av. Goias, 3400 - Barcelona, São Caetano do Sul - SP Cep. 09050-050 - Brasil

3. Doutora em Administração pela Universidade de São Paulo, Faculdade de Economia, Administração e Contabilidade. Professora da Universidade Municipal de São Caetano do Sul - USCS [mromeiro@uscs.edu.br] Endereço da autora: Av. Goias, 3400 - Barcelona, São Caetano do Sul - SP Cep. 09050-050 - Brasil 


\section{ABSTRACT}

This paper is part of a large study to assess the adequacy of the use of multivariate statistical techniques in theses and dissertations of some higher education institutions in the area of marketing with theme of consumer behavior from 1997 to 2006. The regression and conjoint analysis are focused on in this paper, two techniques with great potential of use in marketing studies. The objective of this study was to analyze whether the employment of these techniques suits the needs of the research problem presented in as well as to evaluate the level of success in meeting their premisses. Overall, the results suggest the need for more involvement of researchers in the verification of all the theoretical precepts of application of the techniques classified in the category of investigation of dependence among variables.

Key words: Quantitative methods. Analysis of multiple regression. Analysis joint. Consumer behavior.

\section{RESUMEN}

Este artículo forma parte de un amplio estudio que evalúa la adecuación del uso de técnicas estadísticas multivariantes en las tesis y disertaciones de algunas instituciones de educación superior en el área del marketing, en lo referente a la temática del comportamiento del consumidor entre 1997 y 2006. Este artículo trata de las técnicas de análisis de regresión y de análisis conjunto, dos técnicas con gran potencial de uso en estudios de marketing. Este trabajo tuvo como objetivo analizar si el uso de estas técnicas se ajustaba a las necesidades de los problemas de investigación que surgen en las tesis y disertaciones, y evaluar el grado de acierto en la observancia de sus premisas. En general, los resultados señalan la necesidad de intensificar el comprometimiento de los investigadores en la verificación de todos los preceptos teóricos de aplicación de estas técnicas clasificadas en la categoría de investigación de dependencia entre variables.

Palabras clave: Métodos cuantitativos. Análisis de regresión múltiple. Análisis conjunto. Comportamiento del consumidor.

\section{INTRODUÇÃO}

A ênfase na exigência de qualidade da produção científica nacional em Administração tem ocorrido mais intensamente a partir da década de 1990: Administração Pública (MACHADO-DASILVA et al., 1989); Finanças (LEAL et al., 2003); Marketing (BOTELHO; MACERA, 2001; POWERS et al., 1998; VIEIRA, 1998); Métodos Quantitativos (BREI; LIBERALI, 2004); Operações (ARKADER, 2003), Organizações (BERTERO; KEINERT, 1994; BERTERO; CALDAS, WOOD JÚNIOR, 1999; MACHADODA-SILVA; CUNHA; AMBONI, 1990; RODRIGUES FILHO, 2002; VERGARA; CARVALHO JUNIOR, 1995), Pesquisa em Administração (MARTINS, 1994; PERIN et al., 2002; TORRES, 2000); Recursos Humanos (CALDAS; TONELLI; LACOMBE, 2002; ROESCH et al., 1997; TONELLI et al., 2003), Sistemas de Informação (HOPPEN et al., 1998).

Aspectos epistemológicos e metodológicos são os eixos orientadores na avaliação crítica da qualidade da produção acadêmica nacional. Mas, na literatura pesquisada, há ainda poucos estudos críticos discorrendo sobre questôes metodológicas.

Em estudo sobre a Epistemologia da Pesquisa em Administração, Martins (1994, p. 6566) evidenciou que as dissertaçóes e teses apresentadas na Faculdade de Administraçáo, Economia e Contabilidade da Universidade de São Paulo (FEA/USP), Faculdade de Economia, Administração, Contabilidade e Atuária da Pontifícia Universidade Católica de São Paulo (FEA/PUC) e Escola de Administração de São Paulo (EASP/ FGV), entre os anos de 1980 e 1993, abusavam da utilização do instrumental dos métodos quantitativos de forma bastante superficial dado o nível de sofisticação dessas técnicas. O autor alertou ainda para a despreocupação para com as premissas teóricas de aplicação da maior parte dos métodos.

Para Sánchez Gamboa (1987, p. 17), a importância da análise da produção científica, amparada nas competências metodológicas justifica-se, visto que a sua questão é de fundamental e decisiva importância para o desenvolvimento e resultados da pesquisa científica. 
Conforme o número de variáveis focalizadas simultaneamente nas análises quantitativas, têm-se os seguintes contextos: univariado (enfoque analítico de apenas uma variável por vez), bivariado (enfoque simultâneo de duas variáveis) e multivariado (enfoque simultâneo de três ou mais variáveis). $\mathrm{O}$ uso crescente de técnicas de análise multivariada decorre da contribuição que este contexto pode oferecer à análise quantitativa dos dados para a produçáo científica e do fato de que, nas últimas décadas, vários pacotes computacionais estatísticos se aperfeiçoaram sobremaneira no sentido de tornar seus conteúdos distantes das complexidades matemáticas, próprias dos mesmos pacotes.

A evolução tecnológica notória nos softwares estatísticos tem proporcionado maior facilidade na operacionalização das ferramentas de análise multivariada, contribuindo para a diminuiçáo de barreiras para os usuários e atendendo adequadamente a demanda dos cientistas das ciências sociais aplicadas, entre elas a Administração. Entretanto, tem-se constatado a ocorrência de erros de aplicação, seja na inadequação dos objetivos de uso das ferramentas com os objetivos propostos na pesquisa, seja na violação de premissas de aplicação das técnicas. Há situaçôes em que o pesquisador apenas exercita o emprego de uma técnica e distancia-se de seu problema de pesquisa e do alcance dos objetivos inicialmente traçados.

Uma das áreas da Administração que mais utiliza o método quantitativo, especialmente a análise multivariada, é a área de Marketing. Principalmente pela necessidade de se conhecer o mercado consumidor busca-se a mensuração das opinióes, atitudes, preferências, perfil e outras características dos consumidores (MALHOTRA, 2001).

Os estudos em Marketing, especialmente na temática do Comportamento do Consumidor, vêm se utilizando sobremaneira das ferramentas de análise multivariada. Para Milagre (2001, p. 74), o uso da técnica multivariada tornou-se mais comum a partir do momento que os acadêmicos e profissionais de Marketing passaram a aplicá-la em estudos sobre a preferência e satisfação do consumidor, bem como o seu perfil e comportamento de compras. Martins (1994, p. 66) afirma que os métodos quantitativos são, em Administração, mais aplicados pelos autores de pesquisa nas áreas de Marketing, Produção e Finanças.

Nesse sentido, neste estudo pretende-se aprofundar a discussão e oferecer subsídios à reflexão na temática dos Métodos Quantitativos, especialmente sob o recorte de suas aplicaçóes nos estudos em Marketing sobre o Comportamento do Consumidor.

Entre as técnicas estatísticas multivariadas, a análise de regressão e a análise conjunta destacam-se pela ampla possibilidade de aplicação em Marketing e por pertencerem à mesma categoria de técnicas: investigação da dependência entre variáveis (JOHNSON; WICHERN, 1998, p. 2).

A análise de regressão é uma técnica com grande aplicação na área de Marketing, sobretudo pelo fato de possibilitar a identificação da relação entre variáveis e o dimensionamento do potencial de mercados. A análise conjunta, por sua vez, é uma técnica estatística multivariada que permite determinar a estrutura de preferência do consumidor a partir de avaliaçóes globais de diferentes cenários de produtos ou serviços.

Tendo em vista a relevância destas técnicas no campo de marketing, decidiu-se selecionálas para foco de análise neste trabalho.

Neste contexto, os objetivos a serem alcançados neste estudo são:

a) identificar a intensidade de uso das técnicas multivariadas na área de Marketing, temática do Comportamento do Consumidor, tendo como unidade de análise dissertaçóes e teses, do período 1997-2006, de duas universidades públicas: Faculdade de Economia, Administração e Contabilidade da Universidade de São Paulo e Escola de Administração da Universidade Federal do Rio Grande do Sul (EA/UFRGS);

b) identificar o nível de adequação do uso das técnicas de análise de regressão e análise conjunta aos problemas de pesquisa apresentados nestas dissertaçôes e teses selecionadas;

c) identificar fontes de erros da aplicação das técnicas de análise de regressão e análise 
conjunta, a partir do não atendimento às suas premissas básicas, no conjunto da produção científica examinada.

A seleção desses Programas de Pós-Graduação como público-alvo desse estudo deveu-se à necessidade de delimitação, dada a dificuldade operacional de um levantamento amostral representativo da produção nacional, principalmente pela indisponibilidade de material em base de dados on-line por uma parcela importante das Instituições do país.

Nesse sentido, optou-se inicialmente por um recorte focado nos Programas de Pós-Graduação de alta performance na última avaliação da Coordenação de Aperfeiçoamento de Pessoal de Nível Superior (CAPES). Registre-se que a opção por esse critério não se motivou pela suposição de que os programas com avaliação menos positiva, abaixo da nota 6 , tratariam as técnicas em estudo de forma mais ou menos corretas. A avaliação da CAPES aponta três Instituiçôes com nota 6: a FEA/USP, a EA/UFRGS e a EASP/FGV.

Um segundo critério deu-se devido à necessidade de garantir a maior homogeneidade possível da população alvo, já que não é objetivo específico desse estudo a comparação entre Instituiçóes. Nesse sentido, a seleção levou em conta a natureza da Instituição: pública ou privada. Considerando que entre as três citadas, duas são públicas, optou-se pela análise das dissertaçóes e teses das Instituiçôes públicas: FEA/USP e EA/ UFRGS.

Destacam-se alguns fatores restritivos na abrangência dessa investigação:

a) delimitação do público alvo: os resultados desse estudo são apenas válidos para as dissertaçóes e teses da FEA/USP e da EA/ UFRGS, de 1997 a 2006;

b) as informaçôes sobre o atendimento às premissas da análise de regressão e análise conjunta são baseadas nos relatos dos autores de cada dissertação ou tese sob análise, que, evidentemente, podem conter uma descrição subestimada ou superestimada do que foi realizado.

\section{FUNDAMENTAÇÃO TEÓRICA}

Nesta seção haverá uma introdução sobre análise multivariada, seguida por uma apresentação geral de técnicas multivariadas, finalizando com considerações sobre a análise de regressão e a análise conjunta.

\section{I Análise multivariada}

A análise multivariada permite estudar e evidenciar as ligaçôes, as semelhanças e diferenças existentes entre todas as variáveis envolvidas no processo Bouroche e Saporta apud Trivelloni e Hochheim (1998).

Segundo Steiner (1995), a necessidade de entender a relação entre diversas variáveis aleatórias faz da análise multivariada uma metodologia com grande potencial de uso.

Para Lourenço e Matias (2001), por um lado, as técnicas estatísticas multivariadas são mais complexas do que aquelas da estatística univariada. Por outro lado, apesar de uma razoável complexidade teórica fundamentada na matemática, as técnicas multivariadas, por permitirem o tratamento de diversas variáveis ao mesmo tempo, podem oferecer ao pesquisador um material bastante robusto para a análise dos dados da pesquisa.

Conforme Hair et al. (2005), a análise multivariada auxilia na formulação de questóes relativamente complexas de forma específica e precisa, possibilitando a condução de pesquisas teoricamente significativas.

\subsection{Técnicas estatísticas de análise multivariada}

A escolha dos métodos e tipos de análises empregadas nos trabalhos científicos deve ser determinada pelo problema de pesquisa. Nesse sentido, Johnson e Wichern (1998, p. 2) propóem uma classificação dos objetivos para atendimento do problema em cinco categorias.

O passo seguinte para a escolha da técnica de análise multivariada, após considerar os objetivos do problema de pesquisa é verificar o tipo 


\begin{tabular}{|l|l|}
\hline \multicolumn{1}{|c|}{ Classificação } & \multicolumn{1}{|c|}{ Técnicas relacionadas } \\
\hline \multicolumn{1}{|c|}{$\begin{array}{l}\text { Investigação da dependência entre as variáveis } \\
\text { Todas as variáveis são mutuamente independentes ou uma ou mais variáveis são } \\
\text { dependentes de outras. }\end{array}$} & $\begin{array}{l}\text { Análise Discriminante } \\
\text { Análise de Regressão } \\
\text { Correlação Canônica } \\
\text { Regressão Logística } \\
\text { Análise Conjunta } \\
\text { MANOVA }\end{array}$ \\
\hline $\begin{array}{l}\text { Predição } \\
\text { As relações entre as variáveis devem ser determinadas com o objetivo de de } \\
\text { predizer o valor de uma ou mais variáveis com base nas observações de outras } \\
\text { variáveis. }\end{array}$ & $\begin{array}{l}\text { Análise Discriminante } \\
\text { Análise de Regressão } \\
\text { Análise de Regressão Logística }\end{array}$ \\
\hline $\begin{array}{l}\text { Construção de hipóteses e testes } \\
\text { Hipóteses estatísticas específicas, formuladas em termos de parâmetros da } \\
\text { população multivariada, são testadas. Isto pode ser feito para validar premissas } \\
\text { ou para reforçar convicções prévias. }\end{array}$ & $\begin{array}{l}\text { Modelagem de Equaçães Estruturais } \\
\text { Análise Fatorial Confirmatória }\end{array}$ \\
\hline $\begin{array}{l}\text { Redução dos dados ou simplificação estrutural } \\
\text { O fenômeno em estudo é representado de um modo tão simples quanto possível } \\
\text { sem sacrificar informações importantes. }\end{array}$ & Análise Fatorial Exploratória \\
\hline $\begin{array}{l}\text { Agrupamento de objetos ou variáveis } \\
\text { Grupos de objetivos ou variáveis "similares" são criados com base nas medidas } \\
\text { características. }\end{array}$ & $\begin{array}{l}\text { Análise de Conglomerados } \\
\text { Análise de Correspondência } \\
\text { Escalonamento Multidimensional }\end{array}$ \\
\hline
\end{tabular}

Quadro 1 - Categorias dos objetivos das técnicas estatísticas de análise multivariada.

Fonte: Adaptado de Johnson e Wichern (1998, p. 2).

de relação examinada, número de variáveis dependentes e tipo de escala utilizada.

Sobre o tipo de relação, as técnicas são classificadas como de dependência ou de interdependência. Na primeira situação uma ou mais variáveis (variáveis dependentes) podem ser explicadas ou preditas por outras (variáveis independentes). $\mathrm{Na}$ segunda todas as variáveis são analisadas simultaneamente, sem a orientação de dependência ou independência.

Sobre o tipo de escala utilizada, pode-se generalizar a classificação teórica dessas escalas de mensuração em dois grandes grupos: variáveis métricas e variáveis não métricas.

\subsection{Análise de regressão e análise conjunta}

A seguir, apresentam-se os principais conceitos e premissas das técnicas focalizadas neste estudo.

\subsection{Análise de regressáo}

A terminologia "regressão" foi proposta pela primeira vez por Francis Galton, 1885, em estudo em que demonstrou que a altura dos filhos não tende a refletir a altura dos pais, mas tende a regredir para a média da população (MAROCO, 2003).

O modelo clássico de regressão linear baseia-se na associação entre uma variável dependente Y e uma coleção de variáveis preditoras $\mathrm{x}_{1}$, $\mathrm{x}_{2} \ldots \ldots, \mathrm{x}_{\mathrm{r}}$.

Sobre o objetivo de aplicação da técnica de análise de regressão em função de inúmeros objetivos "secundários" possíveis, faz-se necessário sublinhar que o objetivo mais geral dessa técnica é a avaliação do relacionamento entre variáveis.

As premissas subjacentes ao uso da análise de regressão são as seguintes:

- ausência de observaçóes atípicas: as observaçóes atípicas ou extremas (outliers) são aquelas substancialmente diferentes das outras. Podem ser identificadas no nível univariado, bivariado ou multivariado.

Alguns fatores podem causar a presença de observaçóes atípicas univariadas, alterando o resultado de análise e causando violaçóes de normalidade: erro de entrada de dados ou na codificação; evento extraordinário; observaçôes extraordinárias para os quais o pesquisador náo tem explica- 


\begin{tabular}{|c|c|c|}
\hline Categoria & Principal objetivo indicado no estudo & Fontes \\
\hline \multirow{4}{*}{$\begin{array}{l}\text { - Investigação da } \\
\text { dependência } \\
\text { entre as variáveis } \\
\text { - Predição }\end{array}$} & $\begin{array}{l}\text { Avaliação do relacionamento de uma variável dependente } \\
\text { com diversas variáveis independentes }\end{array}$ & Tabachnick e Fidell (1996) \\
\hline & $\begin{array}{l}\text { Predição de valores de uma variável dependente a partir de } \\
\text { uma coleção de variáveis independentes }\end{array}$ & Johnson e Wichern (1998) \\
\hline & $\begin{array}{l}\text { Identificar se as variáveis independentes explicam uma } \\
\text { variação significativa da variável dependente } \\
\text { Predizer os valores da variável dependente } \\
\text { Determinar a estrutura da relação a partir da equação } \\
\text { matemática que relaciona as variáveis independentes e } \\
\text { dependente } \\
\text { Identificar quanto da variação na variável dependente pode } \\
\text { ser explicado pelas variáveis independentes: intensidade da } \\
\text { relação }\end{array}$ & Malhotra (2001) \\
\hline & $\begin{array}{l}\text { Estabelecer que um grupo de variáveis independentes explica } \\
\text { uma proporção da variação de uma variável dependente a um } \\
\text { nível de significância } \\
\text { Estabelecer a importância de predição relativa das variáveis } \\
\text { independentes (comparando os coeficientes padronizados) }\end{array}$ & Garson (c1998b) \\
\hline
\end{tabular}

Quadro 2 - Objetivos de aplicação da técnica de Análise de Regressão.

ção; observaçóes que representam uma combinação única de valores das variáveis (GARSON, c1998b; HAIR et al., 2005). Sobre os outliers multivariados (casos com valores extremos no conjunto de múltiplas variáveis), há três medidas mais comumente usadas para identificá-los: Distância de Cook, Distância de Mahalanobis e Distância de Leverage;

- ausência de multicolinearidade: segundo Gujarati (2000, p. 318), o termo multicolinearidade foi cunhado por Ragnar Frish em 1934. Originalmente, significa a existência de uma "perfeita" (ou exata) relação linear entre algumas ou todas as variáveis explicativas de um modelo de regressão. Formalmente, o termo multicolinearidade refere-se à existência de mais de uma relação linear exata, ao passo que o termo colinearidade refere-se à existência de uma única relação linear.

Algumas sugestôes para tratamento de multicolinearidade: ignorar se o objetivo é prever; eliminar variáveis; reformular o modelo - usando, por exemplo, a razão entre variáveis; aumentar o tamanho da amostra (GARSON, c1998c; GUJARATI, 2000; HAIR et al., 2005; PESTANA; GAGEIRO, 2000; TABACHNICK; FIDELL, 1996). Testes estatísticos para avaliação da multicolinearidade: Measure of Sampling Adequacy (MSA), Kaiser-MeyerOlkin (KMO), Variance inflation factor (VIF), Índice de condição, Índice de tolerância (GARSON, c1998a; HAIR et al., 2005);

- sensibilidade ao tamanho da amostra: aliado a um correto procedimento de amostragem, o tamanho da amostra deve ser adequado para permitir a generalizaçáo dos resultados, os quais podem ser verificados quanto à significância estatística dos testes; na literatura pesquisada encontramse os seguintes critérios: 104 casos $+\mathrm{m}$, sendo $\mathrm{m}$ o número de variáveis independentes (TABACHNICK; FIDELL, 1996); pelo menos 5 casos para cada variável independente (GARSON, c1998b); pelo menos 40 casos para cada variável independente quando da utilização do método Stepwise (GARSON, c1998b);

- homoscedasticidade: quando a variância dos termos de erro (e) parece constante ao 
longo do domínio da variável preditora, tem-se homoscedasticidade (HAIR et al., 2005); a homoscedasticidade pode ser verificada por meio de gráficos de resíduos (erros) contra os valores reais e contra os valores calculados pela equação; há também alguns testes estatísticos para avaliação da homoscedasticidade, entre eles (GARSON, c1998c; HAIR et al., 2005); Teste de Goldfeld-Quandt; Teste de Park; Teste de Breusch-Pagan-Godfrey; Teste de White's; Teste de Levene para Homogeneidade das Variâncias; Teste de Bartlett's para Homogeneidade das Variâncias; Teste $F$ max; Teste Box's $M$;

- normalidade multivariada: Johnson e Wichern (1998) alertam que, para dados reais, a presença de variáveis com distribuição normal multivariada exata dificilmente ocorre. Nesse sentido, a densidade normal é frequentemente uma aproximação útil à verdadeira distribuição da população; há poucos métodos disponíveis para testar a normalidade multivariada (SHARMA, 1996, p. 380); nos softwares estatísticos LISREL e EQS é possível obter o índice de Mardia, que é baseado nas funçōes de Skewness e Kurtosis; valores menores que três dessa estatística sugerem a normalidade multivariada;

- linearidade: os modelos lineares preveem valores que se ajustam a uma linha reta, que tem uma mudança com unidade constante da variável dependente em relação a uma mudança constante na variável independente (HAIR et al., 2005); a inspeção simples de gráficos como o diagrama de dispersão (scatterplot), por exemplo, é um método simples e comum para a verificação de linearidade; além disso, a análise de correlação e outros testes mais complexos são disponíveis para a detecção dessa premissa; Eisenbeis (1977) e Huberty (1994) admitem o relaxamento dessa premissa quando a normalidade multivariada é atendida e quando a amostra é razoavelmente grande;

- ausência de erros correlacionados: para Gujarati (2000, p. 413), a implicação do não atendimento a essa premissa ocorre principalmente nos testes de hipóteses utilizados na aplicação da técnica; o autor afirma que há o risco de se considerar um coeficiente como estatisticamente significante, ainda que ele possa não ser significativo; alguns testes para a detecção da presença de autocorrelação: Teste de Geary (ou Teste das Carreiras), Teste de Breush-Godfrey, Teste M de Durbin e Teste de DurbinWatson (teste mais largamente utilizado) (GUJARATI, 2000; HAIR et al., 2005).

O Quadro 3 sintetiza as premissas da técnica de análise de regressão.

\begin{tabular}{|l|l|}
\hline \multicolumn{1}{|c|}{ Premissas } & \multicolumn{1}{c|}{ Fontes } \\
\hline Ausência de dados atípicos & Garson (c1998bc), Hair et al. (2005), Tabachnick e Fidell (1996) \\
\hline Ausência de multicolinearidade & $\begin{array}{l}\text { Garson (c1998b), Gujarati (2000), Hair et al. (2005), Malhotra } \\
\text { (2001), Tabachnick e Fidell (1996) }\end{array}$ \\
\hline Tamanho da amostra & Garson (c1998b), Tabachnick e Fidell (1996) \\
\hline Homoscedasticidade & $\begin{array}{l}\text { Garson (c1998b), Gujarati (2000), Hair et al. (2005), Malhotra } \\
\text { (2001), Tabachnick e Fidell (1996) }\end{array}$ \\
\hline Normalidade multivariada & $\begin{array}{l}\text { Garson (c1998b), Gujarati (2000), Hair et al. (2005), Tabachnick e } \\
\text { Fidell (1996) }\end{array}$ \\
\hline Linearidade & $\begin{array}{l}\text { Garson (c1998b), Gujarati (2000), Hair et al. (2005), Malhotra } \\
\text { (2001), Tabachnick e Fidell (1996) }\end{array}$ \\
\hline Ausência de erros correlacionados & $\begin{array}{l}\text { Garson (c1998b), Gujarati (2000), Hair et al. (2005), Malhotra } \\
\text { (2001), Tabachnick e Fidell (1996) }\end{array}$ \\
\hline
\end{tabular}

Quadro 3 - Premissas subjacentes ao uso da Análise de Regressão. 


\subsubsection{Análise conjunta}

A Análise Conjunta (Conjoint Analysis), denominada também de Análise Paritária e Análise de Preferência, integra o grupo das análises multivariadas mais recentes aplicadas na área de Marketing.

Segundo Artes (1991), a primeira produção científica sobre Análise Conjunta data da década de 1970, por meio de estudos realizados por Green e Rao (1971).

No Brasil, conforme Siqueira (1995), o trabalho mais antigo sobre Análise Conjunta aplicado ao Marketing foi o de Motta e Carneiro (1986).

Hair et al. (2005) conceituam a Análise Conjunta como uma técnica estatística de análise multivariada usada para entender como os indivíduos constroem suas preferências por produtos e serviços.

Sobre os objetivos de aplicação, a Análise Conjunta enquadra-se na categoria investigação da dependência entre as variáveis.

Sobre as premissas subjacentes ao uso da Análise Conjunta, Hair et al. (2005) afirmam que essa técnica tem o menor conjunto restritivo de suposições que envolvem estimações de modelos.

Entretanto, o pesquisador deve cuidar para, assim como em outras técnicas estatísticas multivariadas, que o modelo seja especificado de forma correta antes do delineamento de pesquisa.

\section{METODOLOGIA DE PESQUISA}

Nessa seção serão apresentados os tópicos referentes a: caracterização da população, desenho metodológico da pesquisa empírica, a construção do critério de adequação da aplicação das técnicas de análise de regressão e análise conjunta, instrumento de coleta de dados e variáveis de resultados.

\section{I Caracterização da população-alvo}

A população sob análise contempla as dissertaçôes e teses em Administração, área de Marketing, temática do Comportamento do Consumidor, apresentadas nos Programas de PósGraduação da Faculdade de Economia, Administração e Contabilidade da Universidade de São Paulo e da Escola de Administração da Universidade Federal do Rio Grande do Sul, no período entre 1997 e 2006.

$\mathrm{O}$ universo de interesse foi definido como a dissertação ou tese, com foco em Marketing, especificamente comportamento do consumidor, e utilização das técnicas estatísticas de análise de regressão e/ou análise conjunta como instrumento de solução do problema de pesquisa, apresentada aos Programas de Pós-Graduação em Administração (PPGA’s) das instituiçôes públicas com mais alta avaliação pela CAPES na área de Administração.

A unidade populacional, no entanto, refere-se à aplicação de técnica estatística multivariada nesse universo de interesse, podendo ser mais do que uma aplicação em cada estudo integrante do universo de interesse.

A identificação da população-alvo foi realizada em três etapas:

\begin{tabular}{|l|l|c|}
\hline \multicolumn{1}{|c|}{ Categoria } & \multicolumn{1}{|c|}{ Principal objetivo indicado no estudo } & Fontes \\
\hline \multirow{5}{*}{$\begin{array}{l}\text { Investigação da } \\
\text { dependência entre as } \\
\text { variáveis }\end{array}$} & $\begin{array}{l}\text { Estimar a estrutura de preferência de um consumidor a partir } \\
\text { de uma avaliação geral de um conjunto de alternativas que são } \\
\text { pré-estabelecidas em termos de seus diferentes atributos }\end{array}$ & Green e Srinivasan (1978) \\
\cline { 2 - 3 } & $\begin{array}{l}\text { Obter e analisar, por meio de estimação de modelos, expe- } \\
\text { rimentos cujas variáveis respostas expressem preferências } \\
\text { individuais }\end{array}$ & Artes (1991) \\
\cline { 2 - 3 } & $\begin{array}{l}\text { Determinar a função utilidade suposta pelos indivíduos para } \\
\text { cada atributo e sua importância relativa }\end{array}$ & \multicolumn{1}{|c|}{ Kotler (1995) } \\
\cline { 2 - 3 } & $\begin{array}{l}\text { Definir produtos ou serviços com a combinação ótima de } \\
\text { atributos }\end{array}$ & $\begin{array}{l}\text { Medir o impacto de determinados atributos de um produto na (2005) } \\
\text { preferência do consumidor }\end{array}$ \\
\cline { 2 - 3 }
\end{tabular}

Quadro 4- Objetivo de aplicação da técnica de Análise Conjunta. 
a) seleção dos trabalhos que apresentavam o termo comportamento do consumidor como uma das palavras-chave;

b) leitura dos resumos dos trabalhos não selecionados na primeira etapa, com o objetivo de identificar aqueles que tratavam do comportamento do consumidor, ainda que não apresentassem o termo como palavra-chave e, assim, selecioná-los;

c) exame do conteúdo dos trabalhos selecionados na primeira e na segunda etapa, identificando aqueles com aplicação de alguma técnica de análise multivariada.

O universo de interesse do estudo registrou 196 dissertaçôes e teses sobre Comportamento do Consumidor, sendo 56 dissertaçóes e teses com aplicação de técnicas estatísticas multivariadas, as quais resultaram em 99 aplicações de técnicas estatísticas de análise multivariada, já que cada uma das 56 pôde ter aplicado um ou mais técnicas diferentes.

\subsection{Desenho metodológico da pesquisa}

A construção metodológica da pesquisa empírica foi orientada, inicialmente, pela avaliação do processo de solução do problema gerador de dissertaçōes e teses com aplicação de técnica estatística de análise multivariada, na temática aqui selecionada.

Considerou-se, ainda, o fato de que esta avaliação exige ser orientada por um critério, o qual foi construído dentro desse próprio estudo, não tendo sido submetido a um processo de validação anterior.

Essas duas condições, por si só, remetem esse estudo ao âmbito da pesquisa exploratória, visto que o processo de aprofundamento do entendimento do problema é uma etapa aqui cumprida para subsidiar a construção do critério de avaliação da adequação da aplicação da estatística multivariada aos trabalhos selecionados do período 1997-2006.

Nesse sentido, este estudo insere-se na abordagem quantitativa, utilizando a análise de conteúdo.
O delineamento exploratório para este estudo vai ao encontro da abordagem de Selltiz et al. (1974, p. 60) que evidencia ser uma das finalidades desse método a apresentação de um recenseamento de problemas considerados urgentes por pessoas que trabalham em determinado campo de relaçóes sociais. Exemplifica essa convergência o alerta feito em outros estudos de que a rigidez teórica, explicitadas nas premissas para aplicaçóes das técnicas estatísticas de análise multivariada, parece não ser acompanhada de rigidez empírica, explicitadas nas concessóes feitas pelos pesquisadores, o que, por vezes, pode resultar em conclusões não precisas sobre a solução dos problemas.

A opção pelo uso da técnica de análise de conteúdo foi orientada especialmente pela necessidade primária desse estudo de interpretar a situação problema das dissertações e teses selecionadas com o propósito de identificar o processo de sua solução por meio da seleção de uma técnica estatística de análise multivariada pertinente.

Da mesma forma como encontrado na abordagem de vários autores, entre eles Richardson (1999, p. 221-222) e Rocha e Deusdará (2005, p. 309), também aqui, a identificação precisa da natureza da técnica de análise oscila entre a discussão quantitativa e a qualitativa.

Assim, de um lado é buscada uma objetividade bastante intensa (RICHARDSON, 1999, p. 221) para categorização dos problemas das dissertações e teses, mediante a definição de critérios rígidos de julgamento da solução desses problemas. De outro, o processo geral de avaliaçóes a ser implementado às unidades de análise (dissertações e teses) está contaminado por julgamentos, na medida em que a análise do conteúdo supóe também o exame das características ausentes ou registros parciais do atendimento às premissas da técnica estatística utilizada.

Nesse sentido, algumas definiçóes de análise de conteúdo parecem contemplar o caminho metodológico da coleta de dados, de acordo com propostas encontradas em Janis et al. apud Richardson (1999, p. 222): "Assim, a análise de conteúdo pode ser definida como qualquer técnica: na base de regras explicitamente formuladas e sempre quando os juízos do analista sejam con- 
siderados como relatórios de um observador científico".

Entretanto, a definição encontrada em Bardin (1979, p. 31) propicia a convivência com as duas abordagens, na medida em que inclui em sua definição de análise de conteúdo, a geração de indicadores quantitativos ou náo no processo analítico.

Análise de conteúdo é um conjunto de técnicas de análise das comunicações, visando a obter, por meio de procedimentos sistemáticos e objetivos de descrição dos conteúdos das mensagens, indicadores (quantitativos ou não), que permitam inferir conhecimentos relativos às condiçôes de produção dessas mensagens. (BARDIN, 1979, p. 31).

3.3 A construçáo do critério de avaliação da aplicação das técnicas estatísticas: análise de regressão e/ou análise conjunta

Dois critérios são apresentados a seguir com a finalidade de avaliar o grau de acerto na aplicação das duas técnicas focalizadas neste trabalho.

\subsection{Procedimento de categorizaçáo do pro- blema de pesquisa da unidade de análise: Critério 1}

Embora várias técnicas de análise multivariada tenham sido utilizadas no material analisado, neste artigo serão destacados os resultados correspondentes à pertinência e à adequação do emprego da análise de regressáo e/ou da análise conjunta. A orientação para categorizar o problema de pesquisa de cada unidade de análise (unidade "i" de análise) foi dada pela finalidade ou objetivo teórico de aplicação de diferentes técnicas estatísticas de análise multivariada. Segundo Johnson e Wichern (1998, p. 2), as categorias são sintetizadas em cinco modalidades:

a) redução dos dados ou simplificação estrutural;
b) agrupamento;
c) dependência entre variáveis;
d) predição;
e) formulação de hipóteses e testes.

No caso da análise de regressão e da análise conjunta, o objetivo comum em ambas é a investigação da dependência entre variáveis.

A categoria identificada na unidade "i" de análise foi confrontada com a categoria-objetivo da técnica de análise de regressão e/ou análise conjunta, na condição de tratamento estatístico aplicado para solução do problema. A avaliação feita por meio desse confronto tem um caráter dicotômico, ou seja, foi considerada como uma aplicação adequada das referidas técnicas quando a categoria-objetivo de aplicação das técnicas ajustou-se à categoria do problema de pesquisa da unidade "i" de análise.

\section{3. 2 Procedimento de avaliaçáo do atendimen- to às premissas das técnicas estatísticas: análise de regressáo e/ou análise conjunta: Critério 2}

$\mathrm{O}$ atendimento às premissas das técnicas aqui focalizadas foi decorrente da confirmação de que todas as premissas postuladas pela teoria foram atendidas. A violação de pelo menos uma das premissas, explicitada no conteúdo apresentado na unidade "i" de análise, bem como a não explicitação da situação de cada premissa (atendimento ou não atendimento) resultou na categoria "não atendimento às premissas". Dessa forma, o critério de avaliaçáo do atendimento às premissas é dicotômico: atendimento a todas as premissas das técnicas e não atendimento a pelo menos uma das premissas destas técnicas.

\subsubsection{Avaliação final do nível de adequação do uso da análise de regressáo e/ou da análise conjunta}

O registro do nível de adequaçáo do uso destas técnicas foi feito em três níveis (Figura 1): 
- nível 1: (Não adequação do uso da técnica): não ajuste da categoria-objetivo de aplicação da técnica à categoria do problema de pesquisa da unidade "i" de análise, independentemente do atendimento ou não atendimento às premissas destas técnicas (Não atendimento ao critério “ 1 ”).

- nível 2: ajuste da categoria-objetivo de aplicação das técnicas à categoria do problema de pesquisa da unidade " $i$ " de análise e não atendimento a pelo menos uma das premissas das técnicas ou à não explicitação da situação de cada premissa (atendimento ou não atendimento) no documento da unidade “i” (Atendimento ao critério " 1 ” e não atendimento ao critério “ 2 ”).

- nível 3: ajuste da categoria-objetivo de aplicação das técnicas à categoria do problema de pesquisa da unidade "i" de análise e atendimento de todas as premissas das técnicas, conforme documento da unidade “i”. (Atendimento ao Critério 1 e ao Critério 2).

\subsection{Instrumento de coleta de dados e resultados}

Para a coleta de dados foi desenvolvido um instrumento para registro das ocorrências encontradas em cada unidade “i” de análise.

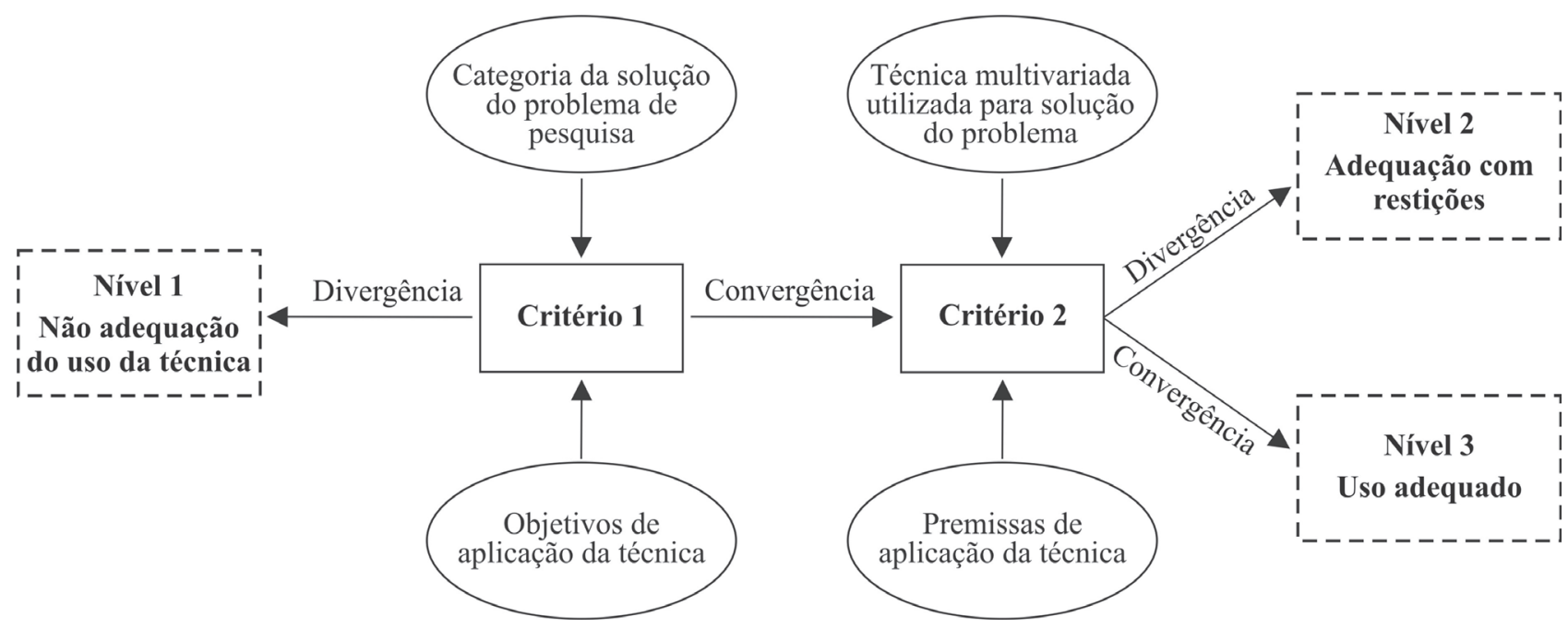

a) identificação numérica das unidades de análise;

b) ano de defesa do trabalho;

c) descrição do problema de pesquisa;

d) categorização do problema de pesquisa a partir dos objetivos de aplicação das técnicas de análise multivariada;

e) identificação da técnica de análise multivariada utilizada no trabalho;

f) aplicação do Critério 1: categorização do problema de pesquisa da unidade de análise;

g) identificação e contagem das premissas atendidas na aplicação da análise de regressão e/ou da análise conjunta, se pelo menos uma destas foi utilizada;

h) aplicação do Critério 2: procedimento de avaliação do atendimento às premissas destas técnicas;

i) avaliação final do nível de adequação do uso destas técnicas.

Os resultados são identificados por meio da ocorrência evidenciada em cada critério. A partir desses resultados, a complementação do plano analítico pressupóe:

a) evidenciar quais técnicas estatísticas de análise multivariada foram mais utilizadas

Figura 1 - Avaliação do nível de adequação da técnica. 
no período de estudo, independentemente da categoria dos problemas de pesquisa;

b) evidenciar as categorias dos problemas de pesquisa que estariam apresentando maior número de ocorrências de aplicação;

c) evidenciar quais premissas são mais negligenciadas no processo de aplicação da análise de regressão e/ou da análise conjunta.

\section{ANÁLISE DOS RESULTADOS}

A primeira etapa de análise dos resultados tratou de apresentar as evidências quanto à intensidade de uso das técnicas multivariadas de modo geral, conforme o objetivo "a".

A segunda parte, conforme o objetivo " $b$ ", tratou do nível de adequação do uso da análise de regressão e/ou da análise conjunta aos problemas de pesquisa das dissertações e teses sob análise.

A terceira parte identificou, a partir do não atendimento às premissas básicas de aplicação destas técnicas, fontes potenciais de erro.

\section{I Resultados relativos ao objetivo "a"}

Entre 1997 e 2006, as dissertações e teses sobre a temática Comportamento do Consumidor, defendidas nos PPGA's da FEA/USP e EA/ UFRGS, apresentaram maior intensidade de uso de técnicas estatísticas multivariadas para atender ao objetivo de reduzir ou simplificar a estrutura de dados coletada (62,5\%), conforme apresentado na figura 2, com a aplicação da técnica de Análise Fatorial Exploratória, única representante dessa categoria.

A categoria de investigação de dependência entre variáveis foi a segunda mais utilizada $(46,4 \%)$. Nesse contexto, seis técnicas de análise multivariada apresentam essa categoria como um dos objetivos de aplicação: Análise de Regressão (15 casos), Análise Discriminante (3 casos), Análise de Variância Multivariada (3 casos), Análise Conjunta (3 casos), Regressão Logística (1 caso) e Correlação Canônica (1 caso).

A Construção de Hipóteses e Testes, exclusivamente representada, neste estudo, pela técnica estatística multivariada de Modelagem de Equações Estruturais somou 18 casos $(32,1 \%)$.

Já a categoria de agrupamento de objetos ou variáveis foi utilizada em 18 estudos (33,9\%), com maior número de casos com uso da técnica de Análise de Conglomerados (12 casos), seguido da técnica de Análise de Correspondência (5 casos) e da técnica de Escalonamento Multidimensional (1 caso).

Cumpre salientar que apenas um dos estudos selecionados utilizou-se de técnica de análise multivariada (regressão logística) com o objetivo de predição de algum fenômeno ou fato, o que possivelmente ocorre mais pela ausência de interesse por problemas de pesquisa na temática de Comportamento do Consumidor do que pela restrição do número de técnicas disponíveis, visto que essa categoria é representada, nesse estudo, por três técnicas de análise multivariada: Análise de Regressão, Análise Discriminante e Regressão Logística.

$$
\begin{aligned}
& \text { Redução dos dados ou simplificação estrutural } \\
& \text { Investigação de dependência entre variáveis } \\
& \text { Construção de testes e hipóteses } \\
& \text { Agrupamento de objetos ou variáveis } \\
& \text { Predição }
\end{aligned}
$$

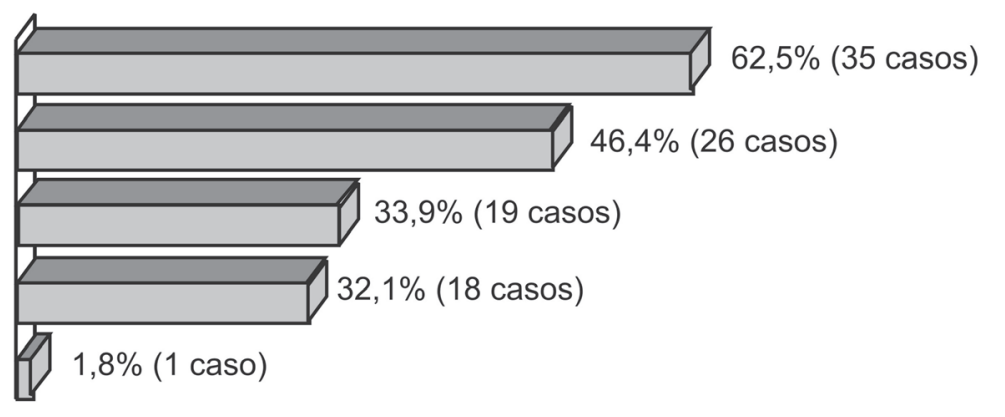

Figura 2 - Categoria dos objetivos de aplicação das técnicas estatísticas multivariadas (base: universo de interesse). 
Abordagem Exploratória do Emprego das Técnicas de Análise de Regressão e Análise Conjunta em Estudos do Comportamento do Consumidor

Tabela 1 - Quantidade de técnicas de análise multivariada diferentes usadas nas dissertaçóes e teses.

\begin{tabular}{l|c|c|c}
\hline \multirow{2}{*}{ Técnica estatística de análise multivariada } & \multicolumn{2}{|c|}{ Nível do estudo } & \multirow{2}{*}{ Total de casos } \\
\cline { 2 - 3 } \cline { 2 - 3 } Uma técnica & Dissertação & Tese & \multirow{2}{*}{$35,7 \%(20$ casos $)$} \\
\hline Duas técnicas & $42,5 \%(17$ casos $)$ & $18,8 \%(3$ casos $)$ & $53,6 \%(30$ casos $)$ \\
\hline Três técnicas & $47,5 \%(19$ casos $)$ & $68,7 \%(11$ casos $)$ & $8,9 \%(5$ casos $)$ \\
\hline Quatro técnicas & $7,5 \%(3$ casos $)$ & $12,5 \%(2$ casos $)$ & $1,8 \%(1$ caso $)$ \\
\hline Média de técnicas & $2,5 \%(1$ caso $)$ & Nenhum caso & 1,8 \\
\hline Mediana de técnicas & 1,7 & 1,9 & 2,0 \\
\hline Moda de técnicas & 2,0 & 2,0 & 2,0 \\
\hline
\end{tabular}

Ainda, a fim de ilustrar o ambiente de uso das técnicas estatísticas multivariadas, registra-se que em $64,3 \%$ dos estudos selecionados, pelo menos duas técnicas são utilizadas para atendimento à solução dos problemas de pesquisa. $\mathrm{O}$ uso mais intenso das técnicas ocorre, em termos relativos, nas teses, com $81,2 \%$ dos casos com aplicação de pelo menos duas técnicas, contra $57,5 \%$ nas dissertaçōes, sugerindo maior sofisticação dos estudos no primeiro grupo.

Quanto às categorias de aplicação da análise de regressão e análise conjunta ao longo do período 1997-2006, deve-se registrar que a categoria de investigação da dependência entre variáveis apresentou tendência de queda no período 1999-2003, voltando a crescer a partir de 2004. Nos anos de 1997 e 1998 não se detectou aplicação de técnica estatística multivariada nas Instituiçôes de ensino alvo desse estudo. A tabela 3 apresenta a evolução do uso das técnicas estatísticas a partir dos objetivos de investigação da dependência entre variáveis e a distribuição específica do emprego da análise de regressão e da análise conjunta.

\subsection{Resultados relativos ao objetivo " $b$ "}

Um resultado positivo deste estudo é a coerência nas dissertações e teses selecionadas quanto à adequação das duas técnicas ao problema de pesquisa.

Assim, pelo critério " 1 ", todas as aplicaçôes das duas técnicas são "adequadas".

\subsection{Resultados relativos ao objetivo "c"}

A técnica de análise de regressão apresentou um baixo índice de premissas atendidas. Mais da metade das dissertações e teses $(66,7 \%)$ não atendeu a nenhuma das premissas exigidas.

Tabela 2 - Distribuição do uso das técnicas com objetivo de investigação da dependência entre variáveis, ao longo do tempo (período 1997-2006).

\begin{tabular}{l|c|c|c|c|c|c|c|c}
\hline & $\mathbf{1 9 9 9}$ & $\mathbf{2 0 0 0}$ & $\mathbf{2 0 0 1}$ & $\mathbf{2 0 0 2}$ & $\mathbf{2 0 0 3}$ & $\mathbf{2 0 0 4}$ & $\mathbf{2 0 0 5}$ & $\mathbf{2 0 0 6}$ \\
\hline $\begin{array}{l}\text { Investigação da dependência } \\
\text { entre variáveis }\end{array}$ & $50 \%$ & $30 \%$ & $38 \%$ & $20 \%$ & $0 \%$ & $25 \%$ & $25 \%$ & $31 \%$ \\
\hline Análise de Regressão & $0 \%$ & $30 \%$ & $39 \%$ & $7 \%$ & $0 \%$ & $10 \%$ & $15 \%$ & $8 \%$ \\
\hline Análise Conjunta & $50 \%$ & $0 \%$ & $0 \%$ & $0 \%$ & $0 \%$ & $10 \%$ & $0 \%$ & $0 \%$ \\
\hline Total & $100,0 \%$ & $100,0 \%$ & $100,0 \%$ & $100,0 \%$ & $100,0 \%$ & $100,0 \%$ & $100,0 \%$ & $100,0 \%$ \\
\hline
\end{tabular}


Com exceção do tamanho da amostra, nenhuma das premissas apresentou mais de $30 \%$ de atendimento, com destaque negativo para homoscedasticidade e linearidade, com atendimento por $13,3 \%$ dos estudos em cada uma dessas premissas.

Apenas um dos estudos que aplicaram a técnica de Análise de Regressão atendeu a todas as premissas exigidas.

Cumpre destacar que, entre os quatro casos de atendimento à premissa de multicolinearidade, dois utilizaram como variáveis independentes os escores fatoriais provenientes de análise fatorial exploratória e os outros dois casos fizeram uso do método stepwise, o qual não exige atendimento à premissa de multicolinearidade. Observou-se, ainda, que, entre os dez casos de não atendimento de nenhuma das premissas, cinco aplicaram a técnica apenas com o intuito de hierarquizar variáveis.

Quanto à análise conjunta, a avaliação é limitada, visto que o único preceito teórico refere-se à especificação do modelo, o qual extrapola a possibilidade de verificação no âmbito da estatística. Portanto, para os três casos de aplicaçáo desta técnica, apenas foi verificada a adequação de seu uso à luz do problema de pesquisa, conforme seção 4.2.

A tabela 3 retrata os índices de atendimento das premissas da técnica análise de regressão. Não foi incluída a análise conjunta nesta tabela pelo fato de nenhuma das premissas teóricas aplicar-se a tal técnica.

4.4 Critério "2" de avaliaçáo dos níveis de adequação do emprego da técnica análise de regressão

O resultado final da avaliação das aplicaçóes encontradas na população-alvo sugere um frágil ambiente operacional de aplicação da técnica análise de regressão, no que se refere especificamente ao atendimento às suas premissas.

O emprego do critério " 2 " do processo de avaliação revela que apenas $6,7 \%$ das aplicaçôes de análise de regressão atendem plenamente às suas premissas.

\subsection{Avaliação final do nível de adequação do uso das duas técnicas}

A avaliação final do nível de adequação do uso da análise de regressão e da análise conjunta pode ser sintetizada pelos índices percentuais obtidos nos três níveis retratados na figura 1 :

- Nível 1: 100\% das aplicaçôes das duas técnicas apresentaram convergência entre a categoria-objetivo de aplicação com a categoria do problema da pesquisa;

Tabela 3 - Atendimento às premissas subjacentes ao uso da técnica regressão múltipla.

\begin{tabular}{|c|c|c|c|}
\hline \multirow{2}{*}{\multicolumn{2}{|c|}{ Premissas }} & \multicolumn{2}{|c|}{ Regressão múltipla } \\
\hline & & $\begin{array}{l}\text { Número de casos } \\
\text { (total de } 15 \text { casos) }\end{array}$ & $\begin{array}{l}\text { \% sobre o total de casos } \\
\text { que utilizaram a técnica }\end{array}$ \\
\hline \multicolumn{2}{|c|}{ Ausência de dados atípicos } & 3 & $20,0 \%$ \\
\hline \multicolumn{2}{|c|}{ Multicolinearidade } & 4 & $26,7 \%$ \\
\hline \multicolumn{2}{|c|}{ Tamanho da amostra } & 15 & $100,0 \%$ \\
\hline \multicolumn{2}{|c|}{ Homoscedasticidade } & 2 & $13,3 \%$ \\
\hline \multirow{2}{*}{ Normalidade } & multivariada & 0 & $0,0 \%$ \\
\hline & univariada & 3 & $20,0 \%$ \\
\hline \multicolumn{2}{|l|}{ Linearidade } & 2 & $13,3 \%$ \\
\hline \multicolumn{2}{|c|}{ Ausência de erros correlacionados } & 3 & $20,0 \%$ \\
\hline \multicolumn{2}{|c|}{ Atendimento a nenhuma premissa } & 10 & $66,7 \%$ \\
\hline \multicolumn{2}{|c|}{ Atendimento a até $\mathbf{5 0 \%}$ das premissas } & 13 & $86,7 \%$ \\
\hline \multicolumn{2}{|c|}{ Atendimento a mais de $\mathbf{5 0 \%}$ das premissas } & 2 & $13,3 \%$ \\
\hline \multicolumn{2}{|c|}{ Atendimento a todas as premissas } & 1 & $6,7 \%$ \\
\hline
\end{tabular}


- Nível 2: 93,3\% das aplicações da técnica análise de regressão atenderam parcialmente as suas premissas;

- Nível 3: 6,7\% das aplicaçóes da técnica análise de regressão apresentaram uso adequado quanto ao atendimento de suas premissas.

\section{CONCLUSÓES}

Nos trabalhados avaliados, as soluçóes para os problemas de pesquisa concentraram-se no emprego de técnicas de redução ou simplificação estrutural dos dados (62,5\% dos trabalhos), seguido de técnicas de investigação de dependência entre variáveis (46,4\% dos trabalhos).

No conjunto dos trabalhos focalizados, o uso de técnicas de investigação da dependência entre variáveis apresentou tendência de queda no período 1999-2003, voltando a crescer a partir de 2004.

Um resultado satisfatório sobre o emprego destas técnicas é que $100 \%$ das suas aplicaçóes apresentaram-se adequadas à resolução dos problemas de pesquisa das dissertaçóes e teses focalizadas nesse estudo.

Quanto à qualidade de aplicação destas técnicas, destaca-se que a verificação de todas as premissas ocorreu apenas em $6,7 \%$ das aplicaçóes de regressão múltipla.

Mesmo que os resultados desse estudo não possam ser extrapolados para a produção acadêmica de outras Instituiçóes ou de outros períodos de tempo, recomenda-se maior cuidado conceitual nas aplicações da técnica de análise de regressão.

A partir dos resultados aqui obtidos, pode-se ressaltar a importância das bancas de qualificação no sentido de alertar os pesquisadores sobre a necessidade desses cuidados, incentivando a prática do atendimento às premissas inerentes às técnicas.

Espera-se que o resultado apurado nesse estudo, relativo ao elevado nível de não adequação do uso da análise de regressão na populaçãoalvo, em função da não verificação de suas premissas, seja um estímulo aos pesquisadores para a busca de um entendimento mais profundo das técnicas a serem empregadas como parte do processo de solução do problema de pesquisa, de forma a minimizar a ocorrência de erros de aplicação.

\section{REFERÊNCIAS}

ARKADER, R. A pesquisa científica em gerência de operações no Brasil. RAE: Revista de Administração de Empresas, Rio de Janeiro, v. 43, n. 1, p. 70-79, jan. 2003.

ARTES, R. Análise de preferência: conjoint analysis. 1991. 198 f. Dissertação (Mestrado em Estatística)-Instituto de Matemática e Estatística, Universidade de São Paulo, São Paulo, 1991.

BARDIN, L. Análise de conteúdo. Lisboa: Ediçóes 70, 1979.

BERTERO, C. O.; KEINERT, T. M. M. A evolução da análise organizacional no Brasil (196193). RAE: Revista de Administração de Empresas, Rio de Janeiro, v. 34, n. 3, p. 81-90, maio/ jun. 1994.

; CALDAS, M. P.; WOOD JÚNIOR, T. Produção científica em administração de empresas: provocações, insinuações e contribuições para um debate local. RAC: Revista de Administração Contemporânea, Curitiba, v. 3, n. 1, p. 147-178, jan./abr. 1999.

BOTELHO, D.; MACERA. Análise meteórica de teses e e dissertaçóes da área de Marketing apresentadas na FGV-EAESP (1974-1999). In: ENCONTRO DA ASSOCIAÇÃO NACIONAL DOS PROGRAMAS DE PÓS-GRADUAÇÃO EM ADMINISTRAÇÃO, 25., 2001, Campinas. Anais... Rio de Janeiro: ANPAD, 2001. 1 CD-ROM.

BREI, V. A.; LIBERALI, G. O uso de modelagem em equações estruturais na área de marketing no Brasil. In: ENCONTRO DE MARKETING, 1., 2004, Porto Alegre. Anais... Rio de Janeiro: ANPAD, 2004. 1 CD-ROM. 
CALDAS, M. P.; TONELLI, M. J.; LACOMBE, B. M. B. Espelho, espelho meu: meta-estudo da produção científica em recursos humanos nos ENANPADs da década de 90. In: ENCONTRO DA ASSOCIAÇÁO NACIONAL DE PROGRAMAS DE PÓS-GRADUAÇÃO EM ADMINISTRAÇÃO, 26., 2002, Salvador. Anais... Rio de Janeiro: ANPAD, 2002. 1 CD-ROM.

EISENBEIS, R. A. Pitfalls in the application of discriminant analysis in business, finance and economics. The Journal of Finance, Cambridge, v. 32, n. 3, p. 875-900, June 1977.

GARSON, G. D. Factor analysis. In:

Statnotes: topics in multivariate analysis. c1998a. Disponível em: <http://faculty.chass.ncsu.edu/ garson/PA765/statnote.htm>. Acesso em: 20 set. 2007.

Multiple regression. In:

Statnotes: topics in multivariate analysis. c1998b. Disponível em: <http://faculty.chass.ncsu.edu/ garson/PA765/regress.htm>. Acesso em: 20 set. 2007.

Testing of assumptions. In:

Statnotes: topics in multivariate analysis. c1998c. Disponível: <http://faculty.chass.ncsu.edu/ garson/PA765/assumpt.htm>. Acesso em 20 jul. 2007.

GREEN, P. E.; SRINIVASAN, V. Conjoint analysis in consumer research: issues and outlook. Journal of Consumer Research, Gainesville, v. 5, p. 103-123, Sept. 1978.

; RAO, V. R. Conjoint measurement for quantifyng judgmental data. Journal of Marketing Research, Chicago, v. 8, p. 335-63, Aug. 1971.

GUJARATI, D. N. Econometria básica. 3. ed. São Paulo: Makron Books, 2000.

HAIR, J. F. et al. Análie multivariada de dados. 5. ed. Porto Algre: Bookman, 2005.
HOPPEN, N. et al. Sistemas de informação no Brasil: uma análise dos artigos científicos dos anos 90. In: ENCONTRO NACIONAL DOS PROGRAMAS DE PÓS-GRADUAÇÃO EM ADMINISTRAÇÃO, 22., 1998, Foz do Iguaçu. Anais... Rio de Janeiro: ANPAD, 1998. 1 CD-ROM.

HUBERTY, C. J. Applied discriminant analysis. New York: John Wiley, 1994.

JOHNSON, R. A.; WICHERN, D. W. Applied multivariate statistical analysis. 4th ed. New Jersey: Printice Hall, 1998.

KOTLER, P. Administração de marketing: análise, planejamento, implantaçáo e controle. São Paulo: Atlas, 1995.

LEAL, R. P. C. et al. Perfil da pesquisa em finanças no Brasil. RAE: Revista de Administração de Empresas, Rio de Janeiro, v. 43, n. 1, p. 91-103, jan. 2003.

LOURENÇO, A.; MATIAS, R. P. Estatística multivariada. Portugal: Instituto Superior de Engenharia do Porto, 2001.

MACHADO-DA-SILVA, C. et al. Produção acadêmica em administração pública: período 19831988. In: REUNIĀO ANUAL DA ASSOCIAÇÃO NACIONAL DE PROGRAMAS DE PÓS-GRADUAÇÃO EM ADMINISTRAÇÃO, 13., 1989, Águas de São Pedro. Anais... Belo Horizonte: ANPAD, 1989. p. 1599-1618.

; CUNHA, V. C.; AMBONI, N. Organizaçóes: o estado da arte da produção acadêmica no Brasil. In: ENCONTRO NACIONAL DOS PROGRAMAS DE PÓS-GRADUAÇÃO EM ADMINISTRAÇÃO, 14., 1990, Belo Horizonte. Anais... Rio de Janeiro: ANPAD, 1990. 1 CD-ROM.

MALHOTRA, N. K. Pesquisa de marketing. Porto Alegre: Bookman, 2001.

MAROCO, J. Análise estatística: com utilização do SPSS. Lisboa: Ediçóes Silabo, 2003. 
MARTINS, G. A. Epistemologia da pesquisa em administração. 1994. 110 f. Tese (Livre-Docência em Controladoria e Contabilidade)-Faculdade de Economia, Administração e Contabilidade, Universidade de São Paulo, São Paulo, 1994.

MILAGRE, R. A. Estatística: uma proposta de ensino para os cursos de administração de empresas. 2001. 160 f. Dissertação (Mestrado em Engenharia de Produção)-Universidade Federal de Santa Catarina, Florianópolis, 2001.

MOTTA, P. C.; CARNEIRO, K. M. A mensuração de utilidades e a concepção de produtos: $O$ extrato bancário. In: REUNIĀO ANUAL DA ASSOCIAÇÃO DE PROGRAMAS DE PÓSGRADUAÇÃO E ADMINISTRAÇÃO, 10., 1986, Florianópolis. Anais... Rio de Janeiro: ANPAD, 1986.

PERIN, M. G. et al. A pesquisa Survey em artigos de marketing nos ENANPADs da década de 90. RIMAR: Revista Interdisciplinar de Marketing, Maringá, v. 1, n. 1, p.44-59, jan/abr. 2002.

PESTANA, M. H.; GAGEIRO, J. N. Análise de dados para ciências sociais: a complementaridade do SPSS. 2. ed. Lisboa: Editora Silabo, 2000.

POWERS, T. et al. Carrer research productivity patterns of marketing academicians. Journal of Business Research, New York, v. 42, n. 1, p. 7586, 1998.

RICHARDSON, R. J. et al. Pesquisa social. 3. ed. São Paulo: Atlas, 1999.

ROCHA, D.; DEUSDARA, B. Análise de conteúdo e análise do discurso: aproximaçóes e afastamentos na (re)construção de uma trajetória. Alea, Rio de Janeiro, v. 7, n. 2, p. 305-322, dez. 2005. Disponível em: <http://www.scielo.br/scielo.php? script=sci_arttext $\&$ pid $=S 1517-106$ X200500020 0010\&lng=en\&nrm=iso $>$. Acesso em: 20 jul. 2007.

RODRIGUES FILHO, J. Estudos críticos em administração no Brasil: classificação da produ- ção de conhecimento sob a ótica da teoria crítica de Jurgen Habermas. 2002. Tese (Administração de empresas)-Universidade Federal da Paraíba, João Pessoa, 2002.

ROESCH, S. M. et al. Tendências da pesquisa em recursos humanos e organizações: uma análise das dissertaçōes de mestrado. In: ENCONTRO NACIONAL DOS PROGRAMAS DE PÓS-GRADUAÇÃO EM ADMINISTRAÇÃO, 21., 1997, Rio das Pedras. Anais... Rio de Janeiro: ANPAD, 1997.

SÁNCHEZ GAMBOA, S. Epistemologia da pesquisa em educação. 1987. 232 f. Tese (Doutorado em Educação) - Faculdade de Educação, Universidade Estadual de Campinas, Campinas, 1987.

SELLTIZ, C. et al. Métodos de pesquisa nas relaçóes sociais. São Paulo: Herder, 1974.

SHARMA, S. Applied multivariate techniques. New York: Wiley, 1996.

SIQUEIRA, J. O. Mensuração da estrutura de preferência do consumidor: uma aplicação de conjoint analysis. 1995. 226 f. Dissertação (Mestrado em Administração)-Faculdade de Economia, Administração e Contabilidade, Universidade de São Paulo, São Paulo, 1995.

STEINER, M. T. A. Uma metodologia para o reconhecimento de padróes multivariados com resposta dicotômica. 1995. 198 f. Tese (Doutorado em Engenharia de Produção)-Universidade Federal de Santa Catarina, Florianópolis, 1995.

TABACHNICK, B. G.; FIDELL, L. S. Using multivariate statistics. 3rd ed. New York: Harper Collins, 1996.

TONELLI, M. et al. Produção acadêmica em recursos humanos no Brasil: 1991-2000. RAE: Revista de Administração de Empresas, Rio de Janeiro, v. 43, n. 1, p. 105-122, 2003.

TORRES, R. R. Estudo sobre os planos amostrais das dissertaçóes e teses em administração 
da Faculdade de Economia, Administração e Contabilidade da Universidade de Sáo Paulo e da Escola de Administraçáo da Universidade Federal do Rio Grande do Sul: uma contribuição crítica. 2000. 246 f. Dissertação (Mestrado em Administração), Faculdade de Economia, Administração e Contabilidade, Universidade de São Paulo, São Paulo, 2000.

TRIVELLONI, C. A. P.; HOCHHEIM, N. Avaliação de imóveis com técnicas de análise multivariada. In: CONGRESSO BRASILEIRO DE CADASTRO TÉCNICO MULTIFINALITÁRIO, 3., 1998, Florianópolis. Anais... Florianópolis: UFSC, 1998. 1 CD-ROM.
VERGARA, S. C.; CARVALHO JUNIOR, D. Nacionalidade dos autores referenciados na literatura brasileira sobre organizaçôes. In: ENCONTRO NACIONAL DOS PROGRAMAS DE PÓS-GRADUAÇÃO EM ADMINISTRAÇÃO, 19., 1995, João Pessoa. Anais... Rio de Janeiro: ANPAD, 1995. 1 CD-ROM.

VIEIRA, G. D. Por quem os sinos dobram? uma análise da publicação científica na área de marketing do ENANPAD. In: ENCONTRO NACIONAL DOS PROGRAMAS DE PÓSGRADUAÇÃO EM ADMINISTRAÇÃO, 22., 1998, Foz do Iguaçú. Anais... Rio de Janeiro: ANPAD, 1998. 1 CD-ROM. 